\title{
Design an Opening Force Measuring Device for Balancing Clips
}

\author{
L. RÓNAI ${ }^{1}$, P. Z. KOVÁCS², V. GÁL ${ }^{3}$ \\ ${ }^{1}$ University of Miskolc, Faculty of Mechanical Engineering and Informatics, Robert Bosch Department of \\ Mechatronics, ronai.laszlo@uni-miskolc.hu \\ ${ }^{2}$ University of Miskolc, Faculty of Mechanical Engineering and Informatics, Institute of Material Science and \\ Technology, metkpz@uni-miskolc.hu \\ ${ }^{3}$ University of Miskolc, Faculty of Mechanical Engineering and Informatics, Institute of Material Science and \\ Technology, metgv@uni-miskolc.hu
}

Abstract. This paper deals with the development of a force measuring device to determine the opening force of different types of balancing clips. These elements can be used in the impellers of ventilators to rebalance the rotor. The measuring device contains a commercially available beam type load cell. The data processing is performed by Arduino Nano development platform with a 24-bit sigma delta A/D converter. The device is well applicable to determine the opening force of the clips in different opening distances.

Keywords: balancing clips, load-cell, microcontroller

\section{Introduction}

Nowadays measuring different environmental signals is essential, therefore sensors are increasingly present not only in industry but also in everyday life. A simple sensor can respond an input physical property and transforms it to electrical signal [1]. There are various types of sensors available in the market, their selection will be determined by the nature of the function.

The importance of force measurement affects many areas, such as home applications, e.g., kitchen scales, room scales, or industrial applications, such as electro-hydraulic press machines [2] or industrial robots to perform force feedback [3]. A load cell [4] contains strain gauges in Wheatstone bridge configuration; therefore, it is a strain gauge-based sensor. Load cells has many types, e.g., beam, S-Beam, pancake, rodend, etc.

In this paper a beam type load cell is used to develop a measuring device, which is capable to determine the opening force of metal balancing clips depending on the opening distance. The balancing clips are located on the impellers of fans to minimize the unwanted vibrations during the operation. This can only happen if the clip keeps its inserted position in the course of operation. This aspect depends most on the clamping force, which can characterize the effectiveness of the clip. At industry conditions there are different types of balancing clips, which have different geometries. 
The data of the measurements can help to review the newly developed geometries and the weights of the balancing clips.

The developed system besides the load cell contains an A/D converter and an Arduino Nano development platform. The designed and manufactured system is protected by utility model rights [5].

The rest of the paper is organized as follows: the developed system, and its components are discussed in Section 1. The program flow chart of the microcontroller, a measurement of an exact type of balancing clips and its results are described in Section 2. The concluding remarks can be found in Section Conclusions.

\section{The developed measurement device}

The measurement device is shown in Figure 1. The load cell is mounted onto an aluminium base with two bolts. The lower holder plate of the balancing clip is placed onto the surface of the load cell. A stand is mounted onto the base to keep an upper holder plate. This plate is connected to a threaded rod, which has $1 \mathrm{~mm}$ pitch. Between the threaded unit and the stand, there is a spring, which ensures the prestressed condition of the rod. A knob can be used to set the discrete positions of the upper holder plate in the course of measurements. The opening distance will be $1 \mathrm{~mm}$ if the knob is rotated with the angle of $360^{\circ}$ due to the pitch of the thread. The applied load cell has $50 \mathrm{~N}$ load capacity, in order to prevent it from unwanted overloading in one direction a set screw is found in the aluminium base element. The contact point of the set screw and the load cell can be adjusted with it.

The beam type load cell consists of four strain gauges in a Wheatstone bridge configuration. Four wires are attached to the A/D converter, which is a HX711 24-bit sigma delta module [6]. This resolution of the converter is required to measure the small changes of the bridge voltage. The converter is connected to the Arduino Nano [7] via serial communication protocol. The Arduino Nano platform contains an ATmega328 microcontroller. The system can be connected to a personal computer with a USB cable.

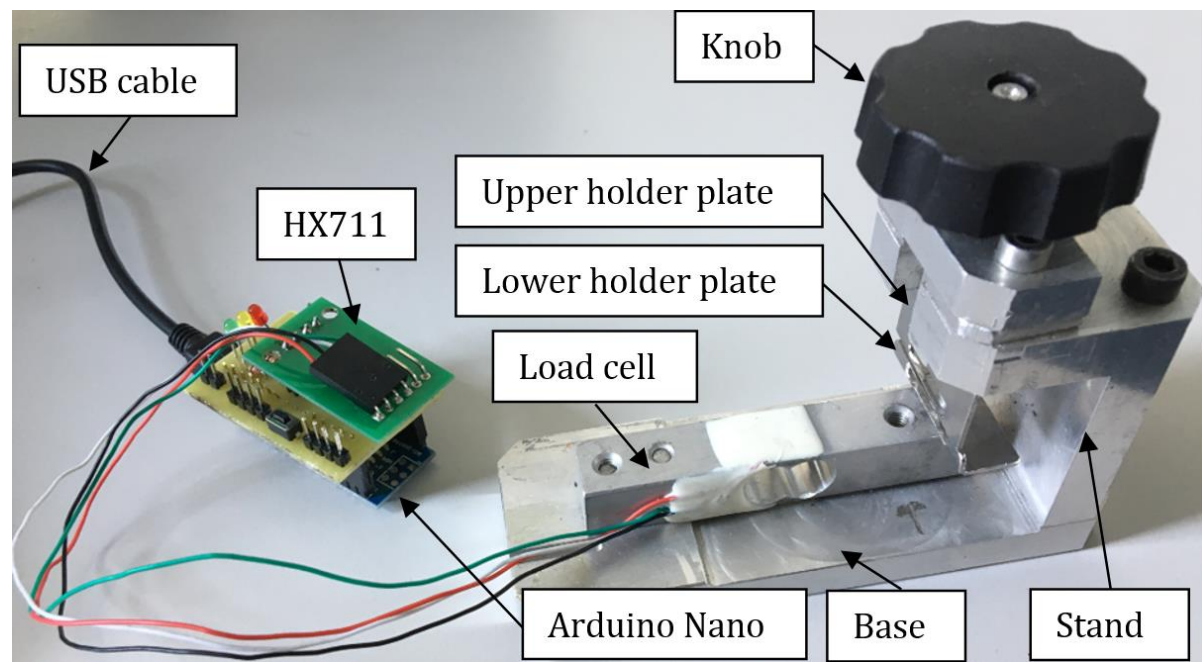

Figure 1. The force measuring device.

The device is well applicable to measure the opening forces of different types of balancing clips. A balancing clip is shown in Figure 2 during a measurement. Post processing the data of the measurements 
is essential to compare the results of the Finite Element Method (FEM) simulations [8]. The block scheme of the developed system is shown in Figure 3.

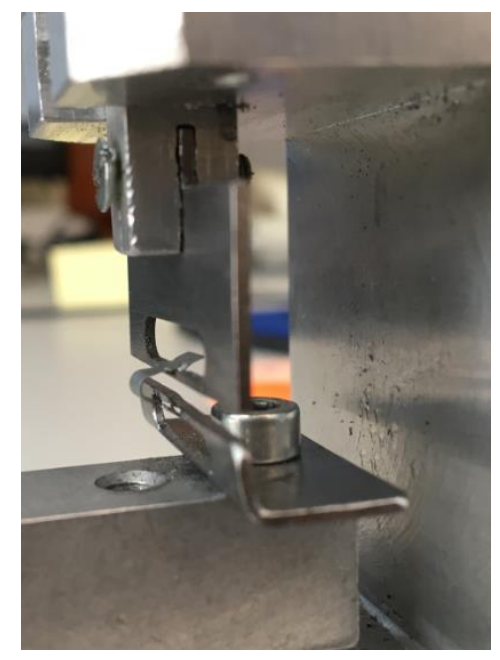

Figure 2. Balancing clip in a discrete opening position.

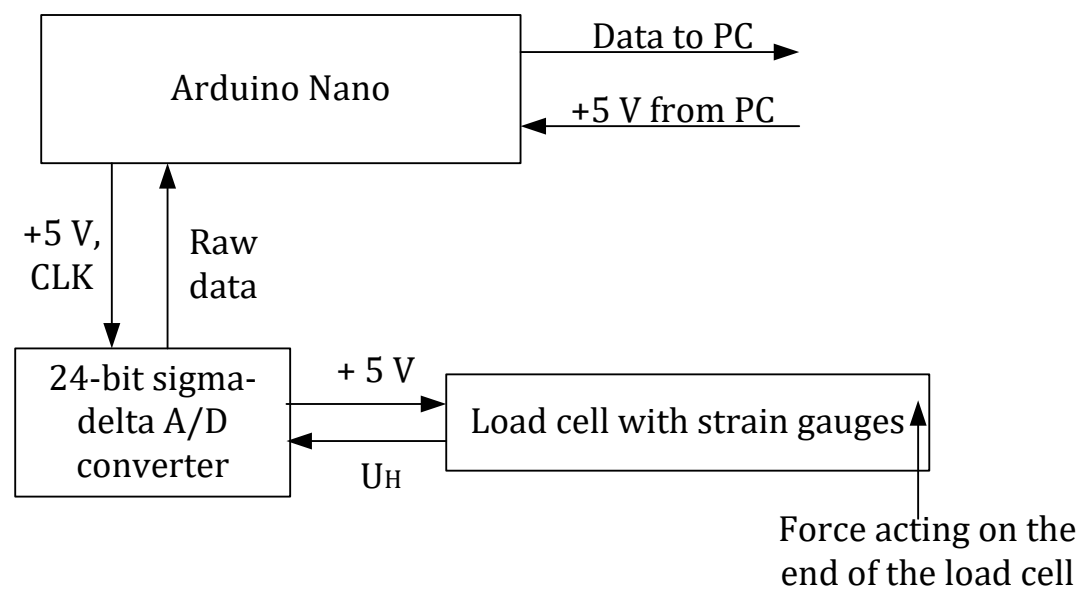

Figure 3. The block scheme of the measurement device.

During the measurement of the balancing clips, the force is acting on the end of the load cell. This force causes a change in the resistance of the strain gauges, therefore the bridge voltage $U_{H}$ is also changed. The A/D converter module measures the bridge voltage of the load cell and digitize it to provide the raw value of the measurement to the microcontroller, which calculates the exact opening force value and transfer it to a PC.

\section{Performing measurements}

The system is programmed in Arduino Integrated Development Environment (IDE) software in C language. A special library was necessary to use the functions of the HX711 A/D converter module. The raw digitized data is sent by the $\mathrm{A} / \mathrm{D}$ converter to the microcontroller.

The first task was to calibrate the load cell to provide accurate force values. The calibration process was performed with the use of known weights to measure its raw values and set the calibration parameter. The parameter $p$ can be calculated with the following formula: 


$$
p=\frac{X_{m}-X_{b}}{m}
$$

where $X_{m}$ is the raw measured value, $X_{b}$ is the raw base value when the system is in rest before the calibration process, and $m$ is the mass of the calibrating weight.

After calibration process the developed program can be implemented to get the actual opening force value of the balancing clips. The incoming data from the microcontroller can be monitored and saved in .csv file format with the use of SerialPlot software. At discrete opening positions, the opening force can be determined from the acquisition data. The flow chart of the developed measurement program can be seen in Figure 4.

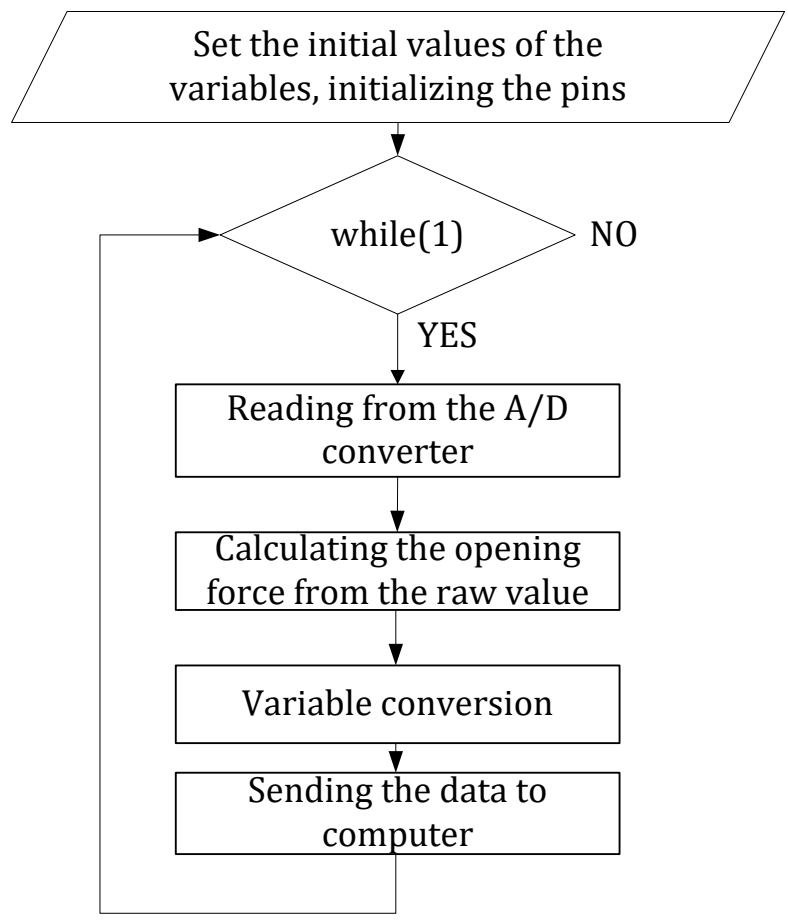

Figure 4. Flow chart of the developed program.

At the first step the initial values of the variables, and the numbers of the pins are defined, e.g., the calibration parameter and the data, serial clock pins of the A/D converter module. The baud rate of the serial communication between the PC and the microcontroller is set in the void setup () section. Thereafter the microcontroller continuously reads the raw value from the A/D converter in the void loop() section, and calculates the opening force, then performs a variable conversion from type double to ASCII representation. Finally, the calculated value is sent to the PC.

One of the measurements is shown in this paper. The opening force values in discrete positions of five balancing clips of the same geometry (see Figure 5) were determined.
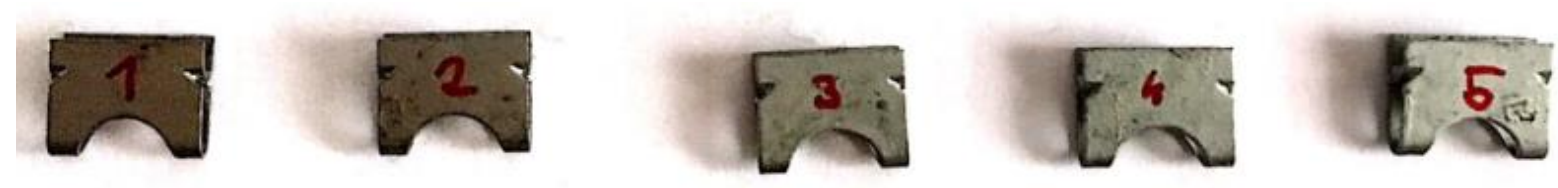

Figure 5. Five balancing clips, which have same geometry. 


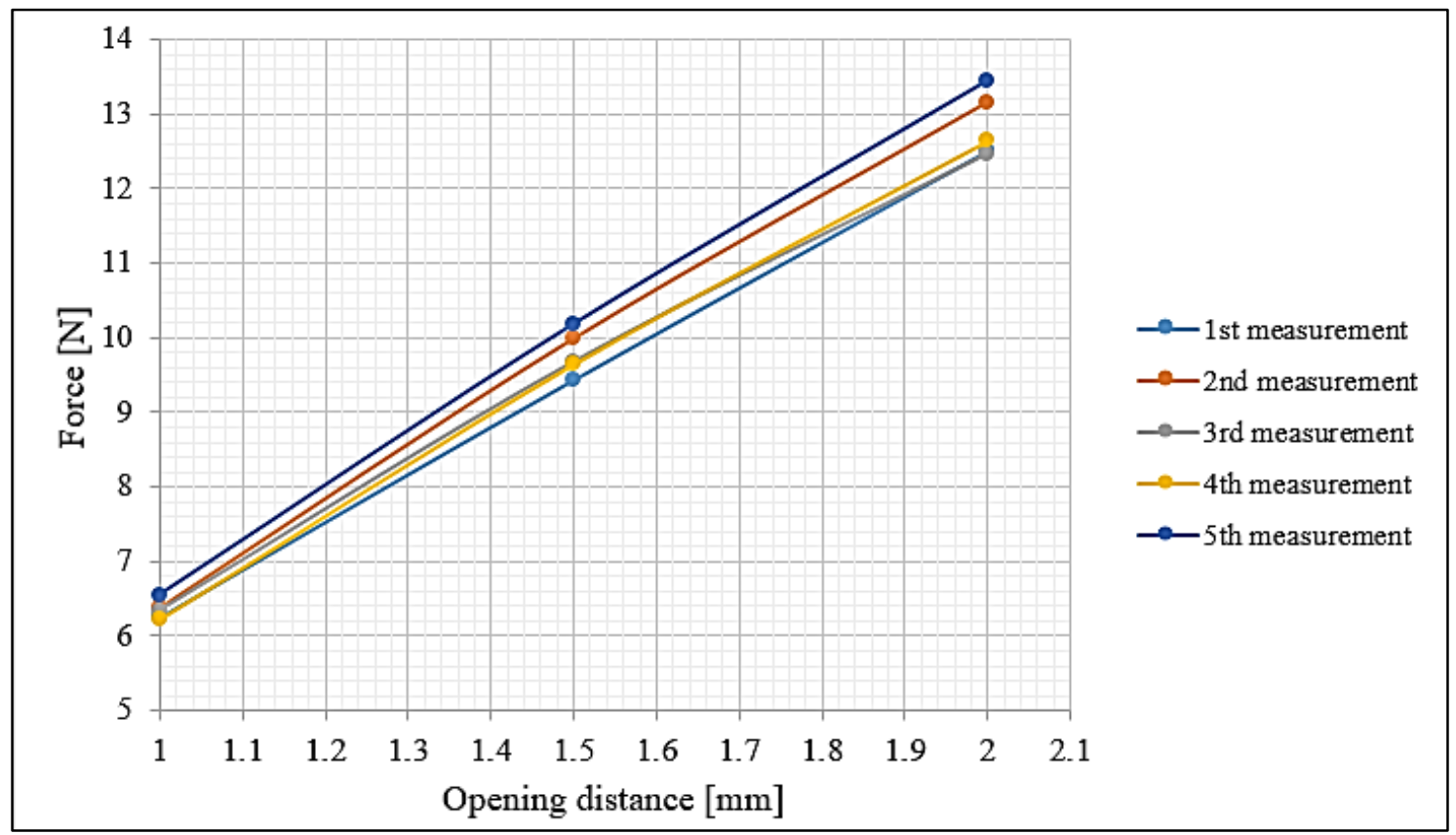

Figure 6. Measurement of the balancing clips.

The results of the measurement of the five balancing clips in discrete opening positions: $1 \mathrm{~mm}, 1.5 \mathrm{~mm}$ and $2 \mathrm{~mm}$ are shown in Figure 6. This data will be well applicable to compare it with FEM simulation results [8], and it can help to reconsider the weight and the shape of the balancing clips.

\section{Conclusions}

In this paper the development of an opening force measurement device for different type balancing clips was detailed. The system has utility model protection rights. The main part of the unit is a beam type load cell, which has four strain gauges in a Wheatstone bridge. In order to measure the change of resistance of the gauges and convert it to electric signal, a 24-bit sigma delta A/D converter and a microcontroller were used. The calibration of the load cell was performed to get accurate results. The program code of the Arduino Nano platform is written in C language. The data of the measurements will be useful to reconsider the geometries of the balancing clips to reduce its weights in the future.

\section{Acknowledgement}

This research work was supported by the European Union and the Hungarian State, co-financed by the European Regional Development Fund in the framework of the GINOP-2.3.4-15-2016-00004 project, aimed to promote the cooperation between the higher education and the industry.

\section{References}

[1] Fraden, J. (2010) 'Handbook of Modern Sensors', 4th edition, Springer New York, Heidelberg, Dordrecht, London, doi: 10.1007/978-1-4419-6466-3. 
[2] Chen, H-M., Yang, G-W, Liao, C-C. (2014) 'Precision Force Control for an Electro-Hydraulic Press Machine', Smart Science, Vol. 2, No. 3, doi: 10.1080/23080477.2014.11665616, pp. 132-138.

[3] Loske, J., Biesenbach R. (2014) 'Force-torque sensor integration in industrial robot control', 15th Int. Workshop on Research and Education in Mechatronics, El Gouna, Egypt, IEEE, doi: 10.1109/REM.2014.6920241.

[4] Muller, I., de Brito, R. M., Pereira, C. E., Brusamarello, V. (2010) 'Load cells in force sensing analysis - theory and a novel application', IEEE Instrumentation \& Measurement Magazine, Vol. 13, No. 1, doi: 10.1109/MIM.2010.5399212, pp. 15-19.

[5] Kovács, P. Z., Rónai, L. (2020) 'Elektromechanikus klipszmérő (Electromechanical clips measurement device) ', utility model protection, patent-roll 5258, (in Hungarian).

[6] AVIA Semiconductor (2016) '24-Bit Analog-to-Digital Converter (ADC) for Weigh Scales', datasheet, pp. 1-9.

[7] Arduino prototyping platform, https://www.arduino.cc/

[8] Kovács, P. Z., Gál, V., Rónai, L., Várkuliné Szarka, Á. (2020) 'Development of Balancing Clips for Ventilator Rotating Elements', GÉP, Vol. LXXI, No. 5-6, pp. 105-109., (in Hungarian) 